\title{
Akutnitrat ermöglicht therapeutisch notwendige Belastung
}

- Dass bei Patienten mit Angina pectoris die medikamentöse Therapie zusammen mit körperlichem Training das Herzinfarkt- und Schlaganfallrisiko besser senken kann als die Implantation eines Stents, wurde $u$. a. in einer großen Studie von Hambrecht et al. 2004 nachgewiesen. „Um im Rahmen eines solchen Trainingsprogramms die therapeutisch notwendige Belastung auch erreichen zu können, ist die Vorabgabe eines schnell wirkenden Nitroglycerins das Mittel der Wahl“, sagte Prof. Rainer Hambrecht, Bremen. Ein bis zwei Hub z. B. von Nitrolingual akut ${ }^{\circledR}$ Spray führen innerhalb von wenigen Minuten zur Vasodilatation der Koronararterien und steigern die Belastbarkeit des Patienten für die nächsten 20 bis 30 Minuten, so Hambrecht. Die Gabe eines Nitrosprays vor körperlicher Belastung wird von nationalen und internationalen Leitlinien empfohlen. Da das $\mathrm{Ni}$ troglycerinspray in diesen Situationen nur unregelmäßig genommen wird, ist eine Toleranzentwicklung nicht zu erwarten.

\section{Seniorengerechte Handhabung}

Die klassische Indikation für Akutnitrate ist die Therapie eines akuten Anginapectoris-Anfalls. Hier ist eine sichere Handhabung genauso wichtig wie die zuverlässige Wirkung des Nitrosprays. Aus diesem Grund hat Dr. Gundolf MeyerHentschel, Saarbrücken, den seniorengerechten Anwenderkomfort verschiedener Nitroglycerin-Spraybehältnisse untersucht. Aus dieser Untersuchung ging Nitrolingual akut ${ }^{\circledR}$ Spray als Testsieger hervor: Die Kappe lässt sich auch mit ge-

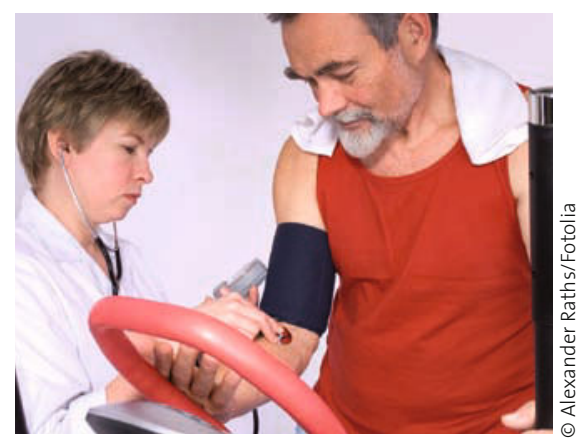

Angina-pectoris-Patienten sollten vor Belastung ein Nitrospray nehmen. ringen Kräften und eingeschränkter Fingerfertigkeit gut öffnen und verschließen, der Sprühmechanismus kann von Älteren mit Einschränkungen in Kraft und Geschicklichkeit gut ausgelöst werden. Die Sprühöffnung hebt sich optisch kontrastreich $a b$ und die rote Farbe der bruchsicheren Plastikflasche erlaubt ein rasches Finden des Medikaments im Notfall.

- Dr. Barbara Kreutzkamp

Ouelle: Fachpressekonferenz, Hamburg, 24.

September 2010 (Veranstalter: Pohl Boskamp)

\section{Kardiale Reanimation}

\section{App für den akuten Notfall}

Einen Service der besonderen Art bietet Pohl Boskamp mit den ,akute Notfälle“ App für das iPhone. Die von Notfallmediziner Dr. Klaus-Gerrit Gerdts, Cuxhaven, erstellte App zur kardialen Reanimation führt den Arzt mit einfachen „Ja-Nein“-Abfragen durch das Menü, das auf Basis der Richtlinien des European Resuscitation Council erstellt wurde.

\section{In der Diabetestherapie zählt nicht nur die $\mathrm{HbA}_{1 c}$-Senkung Vorteil durch geringere Glukosevariabilität}

— Ein ideales Medikament setzt an der Pathogenese der Grundkrankheit an und realisiert die Therapieziele, ohne zusätzlichen Schaden hervorzurufen. Bei den Antidiabetika trifft dies besonders auf DPP-4-Hemmer wie Vildagliptin (Galvus $^{\circledR}$ ) zu, wie Prof. Diethelm Tschöpe, Bad Oeynhausen, darlegte. Neben den Vorteilen seiner Wirkstoffklasse könnten substanzspezifische Eigenschaften zum Therapieerfolg von Vildagliptin beitragen.

Gliptine setzen nicht nur an den Betazellen des Pankreas an, sondern regulieren auch die gestörte Glukagonsekretion. So wird die Inselzelldysfunktion umfassend in den Therapieansatz einbezogen. Auch die dauerhafte effektive $\mathrm{HbA}_{1 c}$-Senkung über bis zu 52 Wochen ist für Vilda- glitpin belegt. Allerdings zähle nicht nur die $\mathrm{HbA}_{1 c}$-Senkung, so Tschöpe, sondern die Gesamtbalance aus Euglykämie und dem Ausbleiben schädlicher Effekte. Ein wichtiger Aspekt für eine gute Wirksamkeit könnte eine geringe Glukosevariabilität sein. In einer aktuellen Studie mit Vildagliptin versus Sitagliptin in Standarddosen fiel das Ergebnis hier zugunsten von Vildagliptin aus.

\section{Metformin wird verträglicher}

An erster Stelle der schädlichen Effekte von Antidiabetika stehen Hypoglykämien. So ist aus der VADT-Studie bekannt, dass sie ein mindestens so großer Prädiktor für die kardiovaskuläre Mortalität sind wie ein Herzinfarkt, sagte Tschöpe.
Mit Vildagliptin ist das Vermeiden von Unterzucker effektiv möglich. Ein zusätzlicher Vorteil ist die Gewichtsneutralität.

Metformin kombiniert mit Vildagliptin (Eucreas ${ }^{\circledR}$ ) hat zudem weniger gastrointestinale (GI) Nebeneffekte. In einer Studie traten mit bis zu $2 \times 1 \mathrm{~g} / \mathrm{d}$ Metformin bei $21 \%$ der Patienten GI-Probleme auf, bei $2 \times 500 \mathrm{mg} / \mathrm{d}$ plus jeweils $50 \mathrm{mg}$ Vildagliptin nur bei $15,4 \%$. In einer weiteren Studie führten $2 \times 1 \mathrm{~g} / \mathrm{d}$ Metformin als Monotherapie bei $11 \%$ der Patienten zu Durchfall, in Kombination mit der Standarddosis Vildagliptin nur bei $6,5 \%$.

\footnotetext{
- Sarah Pampel

Quelle: Symposium und Meet the Expert, EASD-Jahrestagung, Stockholm, 20./22. September 2010 (Veranstalter: Novartis)
} 